\title{
Molten Image Crack Denoising Algorithm Based on the Detection of Connected Domain Area
}

\author{
LIU Xu, YANG Tian-shu, LI Mei ${ }^{\text {}}$, ZHAO Man, ZHAO Yong-ping \\ School of Information Engineering, University of Geosciences, Beijing 100083, China; \\ àmaggielimei@163.com
}

Keywords: Image processing; Crack noise; Connected domain area detection

\begin{abstract}
For the crack noise in molten-image processing of rock deformation experiment, this paper proposes an abnormal value detection method based on connected domain area, through the experiment of comparison with two-dimensional discrete Fourier transform method, this approach can not only completely remove cracks or borders noise, while still maintaining all the information of molten material.
\end{abstract}

\section{Introduction}

A large number of cracks noise in the image is present in the molten image, thus solving the cracks noise of molten image has significant meaning in the quality of the image.

Molten image has two kinds of noise: black internal crack noise from the image and border line noise after partition failed to remove. Therefore, this article attempts to analysis and process the image information from two perspective of frequency-domain and spatial domain respectively, based on two-dimensional spatial Fourier transform. Experiments show that for the cracks noise of molten image, the effect of abnormal value detection method based on connected domain area[1] is better than the two-dimensional discrete Fourier transform[2] method.

\section{Frequency domain-----Two-dimensional discrete Fourier transform}

Image denoising was designed to reduce noise in the image. Reducing noise can be completed in the image space domain, or in the image transform domaim[3]. Denoising methods on spatial domain are much more than on transform domain, so plenty of noise information that spatial denoising method cannot remove, using the image transform domain can often work wonders.

Fourier Transforms in signal processing is one of the most important and widely used transform[4]. For two-dimensional discrete-time signal $\mathrm{f}(\mathrm{x}, \mathrm{y}), \mathrm{x}=0,1, \ldots, \mathrm{X}-1, \mathrm{y}=0,1, \ldots$, Y-1, whose Fourier transform definitions are as follows:

$$
F(u, v)=\frac{1}{X Y} \sum_{x=0}^{X-1 Y Y-1} \sum_{y=0} f(x, y) e^{-j 2 \pi\left(\frac{u x}{X}+\frac{v y}{Y}\right)}
$$

In the above formula: $\mathrm{u}=0,1, \ldots, \mathrm{X}-1, \mathrm{v}=0,1, \ldots, \mathrm{Y}-1, \mathrm{u}$ and $\mathrm{v}$ are called as the spatial frequency, $\mathrm{j}$ is the imaginary unit. The inverse transform definition is as follows:

$$
f(x, y)=\sum_{u=0}^{X-1} \sum_{u=0}^{Y-1} F(u, v) e^{j 2 \pi\left(\frac{u x}{X}+\frac{v y}{Y}\right)}
$$

In the above formula, $\mathrm{x}=0,1, \ldots, \mathrm{X}-1, \mathrm{y}=0,1, \ldots, \mathrm{Y}-1$. Using the fast Fourier transform algorithm can greatly reduce the amount of calculation.

After Fourier transform of the original images and the binary images, respectively, the two pictures use low-pass and high-pass filters. As figures show, using low-pass filter in processing on image of frequency domain can retain its main features, but details are filtered out, so using low-pass filter, image gray value overall changes are not obvious, general information retained, this can be seen from the low-pass filtering restore figure of experiment original image and experiment binary figure, but image become fuzzy, this point can be seen from low- pass partial view. 
Using the high-pass filter in the frequency domain processing of an image, can retain its details, but the main features can be filtered out, so after using the high-pass filter, image gray value changes a lot better as a whole, and this can be seen from the high-pass restore images, but image details are still apparent, and this can be seen from high-pass partial view.

The analysis above shows that, image processing from the frequency domain using filter, while it is possible to reduce the impact of cracks and noises of the border, but noise can't be completely removed, in addition, blurring the molten material, so the frequency-domain denoising method based on Fourier transform in this lab is less effective, it cannot meet the test requirement.

For gray-scale images processing:

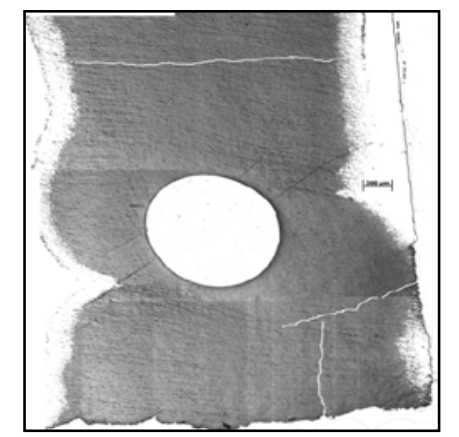

Fig. 1 Experimental original image

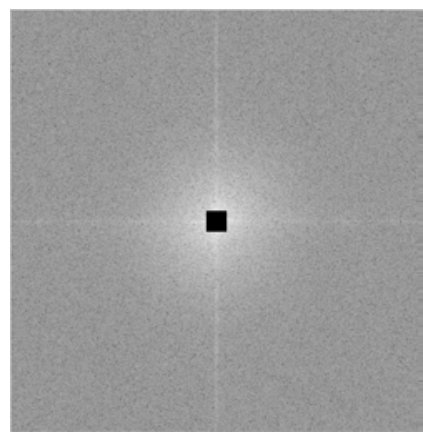

Fig. 4 Frequency domain image after high-pass filtering

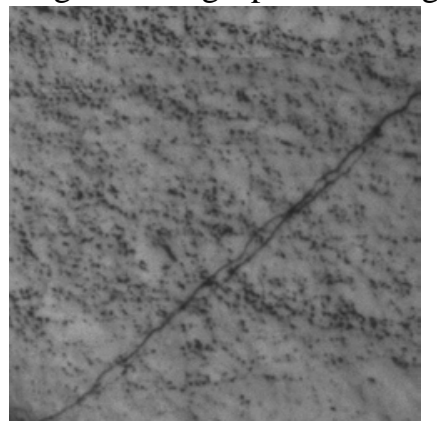

Fig.7 Original image partial view For binary image processing:

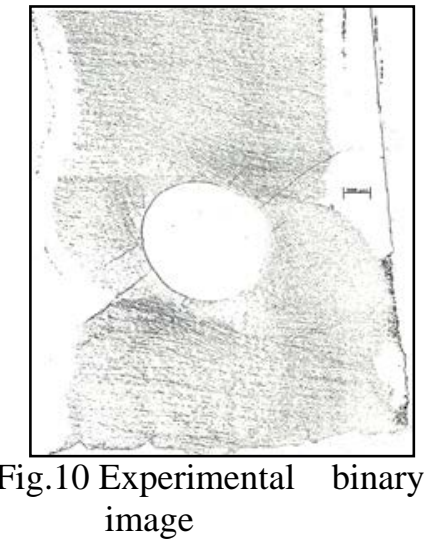

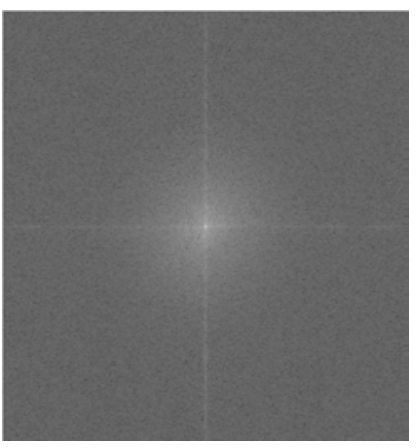

Fig.2 Frequency domain image of original image

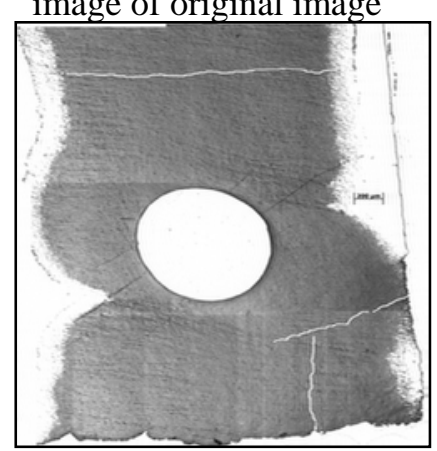

Fig.5 Low-pass filter restore image

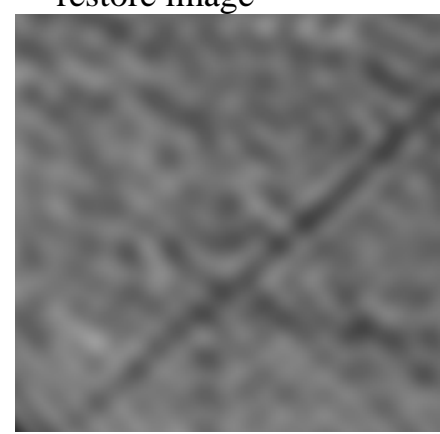

Fig. 8 Low-pass partial view

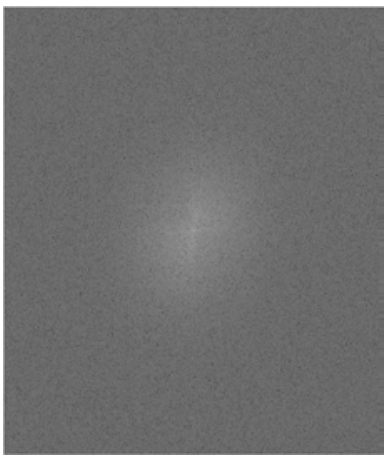

Fig.11 Frequency domain image of binary image

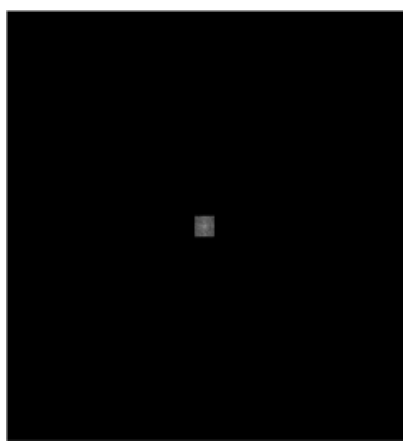

Fig.3 Frequency domain image after low-pass filtering

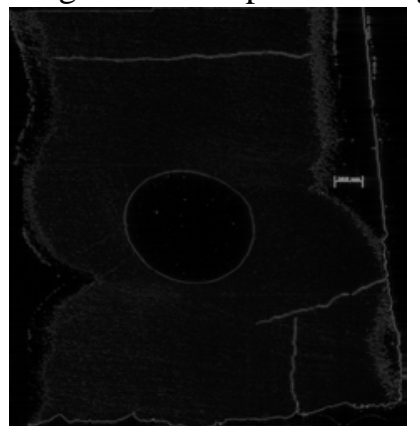

Fig.6 High-pass filter restore image

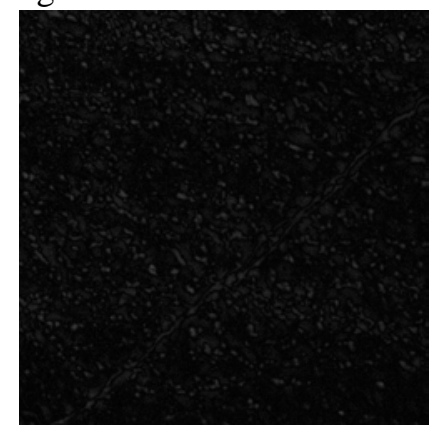

Fig. 9 High-pass partial view

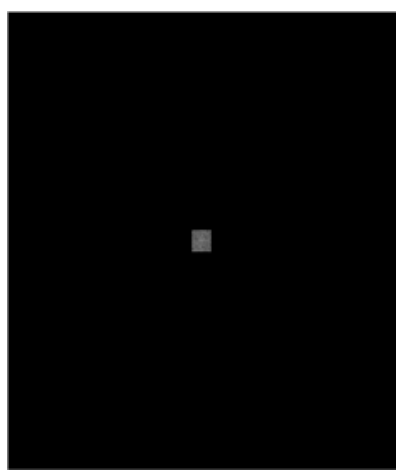

Fig.12 Frequency domain image after low-pass filtering 


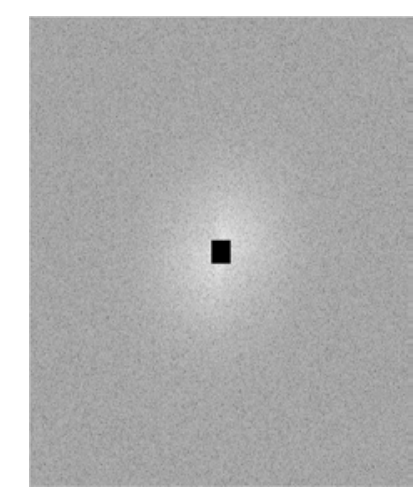

Fig.13 Frequency domain image after high-pass filtering

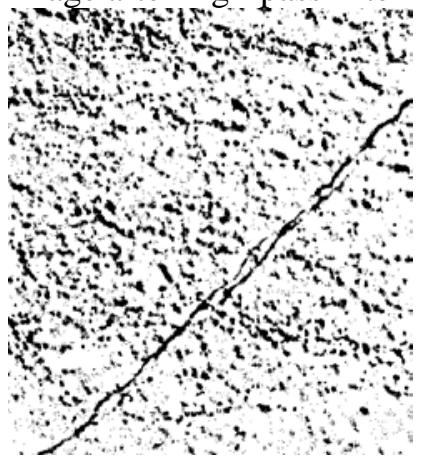

Fig.16 Binary image partial view

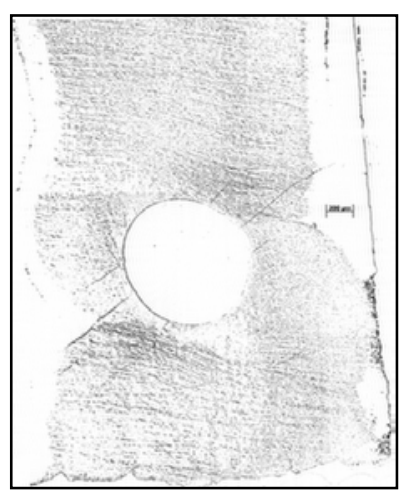

Fig.14 Low-pass filter restore image

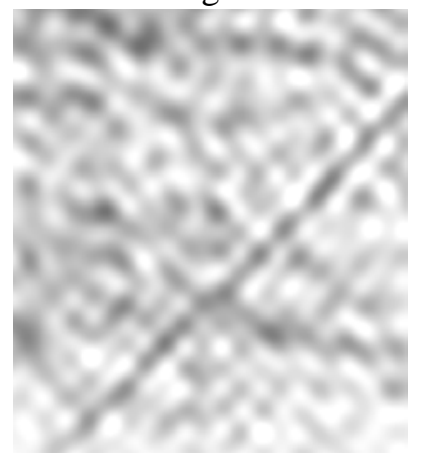

Fig.17 Low-pass partial view

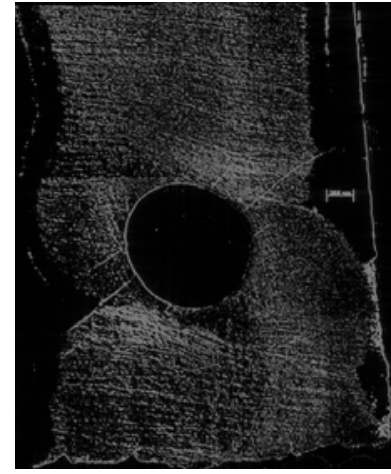

Fig.15 High-pass filter restore image

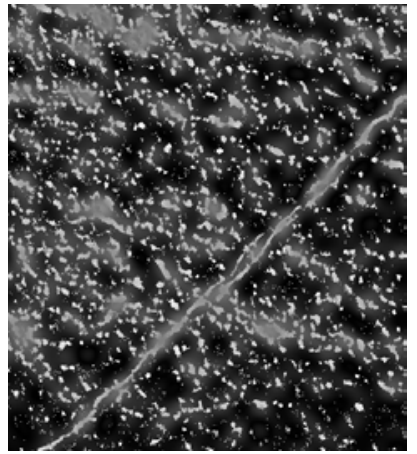

Fig.18 High-pass partial view

\section{Spatial domain------- Outlier detection method for connected domain area[5]}

The analysis can be drawn, the area, which is to remove, of the cracks and boundary line in the image is larger than the molten material and therefore, analyze differences between the cracks and boundary line and the area of molten material to remove noise. Noise removal algorithms are as follows:

Step one: To negate the binary image, melting point is 1 , non-melting point is 0 ;

Step two: The binary value of 1 in the figure of connected domain marked with bwlabel functions in MATLAB[6] and generate a new matrix L;

Step three: For marked matrix L, using regionprops function[6] to obtain areas corresponding to each connected domain;

Step four: Analyze the area of the connected domain with gingham Las algorithm of finding the gross error, to obtain the abnormal area value;

Step five: Find the number of corresponding connected domains through an abnormal value of the area, and remove the corresponding connected domain.

Through the above algorithm, the resulting binary is shown below:

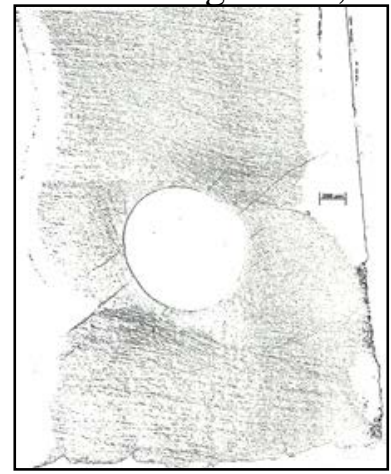

Fig. 19 Noise is not removed binary image

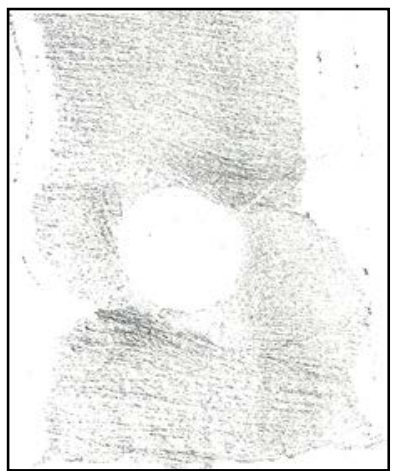

Fig. 20 Noise has been removed binary image

As shown in the figure, through the analyzing and processing algorithm of the area based on spatial domain, the noise of cracks and borders will be completely removed, while still maintaining 
all the information of molten material.

By comparing the spatial domain area detection algorithm and the method of Fourier Transforms and filters in the frequency domain, it is not difficult to find in the picture, for the noise processing in this image, the former method is better.

\section{Summary}

For the cracks noise in the molten image, according to the fact that the area of the cracks noise and boundary line in the image is larger than the molten material, we consider to remove the noise by analyzing differences between the cracks and boundary line, and the area of molten material to remove noise, and propose the abnormal area value detection method based on connected domain area. Comparing with the denoising result of using two-dimensional Fourier transform, the method can be a perfect solution to the cracking noise problems, while still maintaining all the information of the molten material.

\section{References}

[1] HAN Si-qi, WANG Lei. Review of threshold method in image segmentation[J]. Systems Engineering and Electronics,2002,24(6):91-94.

[2] Chen Bing-quan, Liu Hong-li, Meng Fan-bin. Present situation and development direction of digital image processing technology. Jishou University .2009,30 (1): 63-70.

[3] Liu Qing, Wen Zhi-xian, Yang Xiao-ping, Qin Yun-xia. Modern digital image noise filtering technology and its evaluation [J] Automation and Instrumentation, 2012,02: 146-148.

[4] Wang Wen-fa, Yan Yan-bao. Fourier transform analysis feature for image processing. Yanan University 2006,25 (3): 22-24.

[5] LI Qiang. Research on threshold method in image segmentation[J]. Journal of Tongren vocational and technical college,2008,6(6):52-54.

[6] Chen Jie .MATLAB collection (third edition) Beijing: Electronic Industry Press, 2007,1-860. 